\title{
Millimeter observations of planetary nebulae
}

\section{A contribution to the Planck pre-launch catalogue}

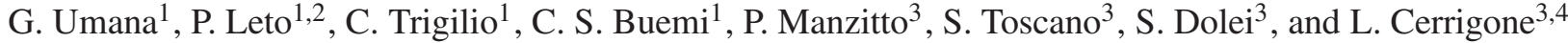 \\ 1 INAF - Osservatorio Astrofisico di Catania, via S. Sofia 78, 95123 Catania, Italy \\ e-mail: Grazia.Umana@oact.inaf.it \\ 2 INAF Istituto di Radioastronomia, Sezione di Noto, C.P. 161, Noto (SR), Italy \\ 3 Universitá di Catania, Dipartimento di Fisica e Astronomia, via S. Sofia 78, 95123 Catania, Italy \\ ${ }^{4}$ Harvard-Smithsonian Center for Astrophysics, Cambridge, MA 02138, USA
}

Received 5 October 2007 / Accepted 18 January 2008

\section{ABSTRACT}

\begin{abstract}
Aims. We present $43 \mathrm{GHz}(7 \mathrm{~mm})$ observations of a sample of radio-bright Planetary nebulae aimed to obtain, together with far-IR measurements (IRAS), reliable estimates of the fluxes emitted in the millimetre and sub-millimetre band, and, therefore, to test their detectability by the forthcoming ESA PLANCK mission. This spectral range, even though very important to constrain the physics of circumstellar environments, is still far from being completely exploited.

Methods. The new millimetre $43 \mathrm{GHz}$ observations were obtained by using the $32 \mathrm{~m}$ INAF-IRA Noto Radiotelescope. To estimate the millimetre and sub-millimetre fluxes, we extrapolated and summed the ionized gas (free-free radio emission) and dust (thermal emission) contributions in this frequency range. By comparison of the derived flux densities to the predicted sensitivity we investigate the possible detection of such source for all the channels of PLANCK

Results. We conclude that almost $80 \%$ of our sample will be detected by PLANCK, with a higher detection rate in the higher frequency channels, where there is a good combination of brighter intrinsic flux from the sources and reduced extended Galactic foregrounds contamination despite poorer instrumental sensitivity. From the new $43 \mathrm{GHz}$, combined with single-dish $5 \mathrm{GHz}$ observations from the literature, we derive radio spectral indexes, which are consistent with optically thin free-free nebula. This result indicates that the high frequency radio spectrum of our sample sources is dominated by thermal free-free, and other emissions, if present, are negligible.
\end{abstract}

Key words. ISM: planetary nebulae: general - radio continuum: general - radio continuum: stars

\section{Introduction}

The PLANCK ESA mission will provide nearly full sky maps over a wide range of frequencies, from 30 to $900 \mathrm{GHz}$. Therefore, even if designed for cosmological studies, the mission will have a profund impact on fundamental physics and Galactic and extragalactic astrophysics. Planck will be sensitive to the millimetre emission from dusty envelopes of stars and we expect the dusty circumstellar envelopes, that characterize the later stages of stellar evolution, to be source of a foreground contamination. When a low or intermediate mass star is approaching the end of its evolution, it goes through a period of heavy mass-loss known as the Asymptotic Giant Branch (AGB) phase. The ejected envelopes are partially condensed in dust grains and completely obscure the central star. Immediately after the AGB evolutionary phase, the mass-loss stops and the stars may become optically visible as the dusty shells disperse (Proto Planetary Nebula, PPN phase). Eventually, once it reaches a temperature of $2-3 \times 10^{4} \mathrm{~K}$, the central star starts to ionize the AGB shell and a Planetary Nebula will form. Dusty envelopes re-radiate the absorbed stellar light showing a clear signature in the far-infrared spectrum, i.e. an IR excess with peculiar IRAS colours. In addition to that, $\mathrm{PNe}$ show a radio continuum due to free-free emission from the fraction of the CSE ionized by the central star.
From a feasibility study, Umana et al. (2006) concluded that a sizable $(\approx 300)$ sample of AGB and post-AGB stars would be detected during the mission and derived estimates for the expected flux densities at various Planck channels. However, the simulations carried out by Umana et al. (2006) on a subsample of PNe rely only on the $1.4 \mathrm{GHz}$ NRAO VLA Sky Survey (NVSS) fluxes, extrapolated to the Planck frequencies. This leads to underestimation of the contribution due to freefree emission as, at this frequency, PNe are often optically thick (Siódmiak \& Tylenda 2001; Luo et al. 2005).

$\mathrm{PNe}$ are among the brightest Galactic radio sources. Some of them could also reach a flux density of Jy level. More than 800 have been detected at least at one frequency and for 200 morphological and spectral information (between 1.4 and $22 \mathrm{GHz}$ ) were obtained. Most of the higher frequency $(22 \mathrm{GHz})$ data were obtained with interferometers (i.e. VLA) while very few singledish, high frequency measurements are available.

In this paper we present new $43 \mathrm{GHz}$, single-dish observations using the $32 \mathrm{~m}$ INAF-IRA Radiotelescope at Noto of a sample of PNe, which are potential foregrounds for PLANCK. The main goal of this project is to obtain reliable estimates of the flux density expected in PLANCK channels, by building and modeling their spectral energy distribution (SED). This in turn will contribute to the compilation of the PLANCK pre-launch catalogue. $43 \mathrm{GHz}$ is one of the observing channels of the forthcoming PLANCK mission. Therefore, at this frequency band, 
Table 1. The selected sample.

\begin{tabular}{|c|c|c|c|c|c|c|c|}
\hline $\begin{array}{l}\text { IAU Name } \\
\text { PN G }\end{array}$ & Other Name & $\begin{array}{l}\text { RA (J2000) } \\
{[\mathrm{h} \mathrm{m} \mathrm{s]}}\end{array}$ & $\begin{array}{c}\operatorname{Dec}(\mathrm{J} 2000) \\
{\left[{ }^{\circ},{ }^{\prime},{ }^{\prime}\right]}\end{array}$ & $\begin{array}{l}\text { IAU Name } \\
\text { PN G }\end{array}$ & Other Name & $\begin{array}{c}\text { RA (J2000) } \\
{[\mathrm{h} \mathrm{m} \mathrm{s}]}\end{array}$ & $\begin{array}{c}\operatorname{Dec}(\mathrm{J} 2000) \\
{\left[{ }^{\circ},{ }^{\prime}\right.}\end{array}$ \\
\hline $000.3+12.2$ & IC 4634 & 170133.6 & -214933.1 & $093.4+05.4$ & NGC 7008 & 210032.7 & +543239.4 \\
\hline $002.4+05.8$ & NGC 6369 & 172920.5 & -234535.0 & $3.5+01.4$ & PN M $1-78$ & 212044.8 & +515327.5 \\
\hline $003.1+02.9$ & PN Hb 4 & 174152.8 & -244209.3 & $096.4+29.9$ & NGC 6543 & 175833.4 & +66 3758.8 \\
\hline $006.7-02.2$ & PN M 1-41 & 180930.6 & -241228.7 & $097.5+03.1$ & PN A66 77 & 213210.2 & +555243.2 \\
\hline $007.2+01.8$ & PN HB 6 & 175507.0 & -214441.0 & $106.5-17.6$ & NGC 7662 & 232553.9 & +423204.7 \\
\hline $008.0+03.9$ & NGC 6445 & 174915.0 & -2000 33.7 & $107.8+02.3$ & NGC 7354 & 224019.9 & +611708.0 \\
\hline $008.3-01.1$ & PN M 1-40 & 180826.0 & -221653.4 & $120.0+09.8$ & NGC 40 & 001301.0 & +723119.6 \\
\hline $009.4-05.0$ & NGC 6629 & 182542.5 & -231211.3 & $130.9-10.5$ & NGC 650-51 & 014219.7 & +513431.7 \\
\hline $009.6+14.8$ & NGC 6309 & 171404.3 & -125437.2 & $138.8+02.8$ & IC 289 & 031019.3 & +611900.4 \\
\hline $.1+00.7$ & NGC 6537 & 180513.1 & -195034.4 & $144.5+06.5$ & NGC 1501 & 040659.3 & +605514.7 \\
\hline & NGC 6578 & 181616.5 & -20 2703.4 & $165.5-15.2$ & NGC 1514 & 916.9 & +304632.0 \\
\hline $011.7-00.6$ & 6567 & & -190435.6 & $166.1+10.4$ & IC 2149 & 5623.9 & +4606 17.4 \\
\hline $020.9-01.1$ & PN 1 & 329.0 & -110 & $173.7+02.7$ & PP 40 & 52.7 & +354218.6 \\
\hline $025.8-17.9$ & 18 & 7.8 & -14( & +02.5 & J 900 & 7.3 & 4727.6 \\
\hline $027.7+00.7$ & $2-45$ & 3921.9 & -041952.6 & $197.8+17.3$ & NGC 2392 & 072910.8 & +2054 41.6 \\
\hline $8-02.6$ & 741 & 0237.0 & -002657.2 & $206.4-40.5$ & NGC 1535 & 041415.8 & -124422.3 \\
\hline $034.6+11.8$ & 6572 & 181206.3 & +065112.4 & $215.2-24.2$ & IC 418 & 052728.2 & -124150.2 \\
\hline $035.1-00.7$ & $2-1$ & 185810.5 & +013657.5 & $221.3-12.3$ & IC 2165 & 062142.8 & -125913.9 \\
\hline $037.7-34.5$ & NGC 7009 & 210410.8 & -112148.5 & $234.8+02.4$ & NGC 2440 & 074155.4 & -181230.5 \\
\hline $039.8+02.1$ & PN K 3-17 & 185618.2 & +0707 26.2 & $254.6+00.2$ & NGC 2579 & 082054.1 & -361300.0 \\
\hline $041.8-02.9$ & NGC 6781 & 191828.1 & +063220.0 & $258.1-00.3$ & Hen 2-9 & 082828.0 & -39 2339.4 \\
\hline $043.1+37.7$ & NGC 6210 & 4429.5 & +234759.9 & $259.1+00.9$ & Hen 2-11 & 083708.1 & -392504.9 \\
\hline $045.7-04.5$ & NGC 6804 & 193135.1 & +09 1330.2 & $261.0+32.0$ & NGC 3242 & 102446.1 & -183832.3 \\
\hline $050.1+03.3$ & PN M 1-67 & 191131.1 & +165132.0 & $294.1+43.6$ & NGC 4361 & 122430.8 & -184704.0 \\
\hline $054.1-12.1$ & NGC 6891 & 201508.9 & +124215.4 & $342.1+10.8$ & NGC 6072 & 161258.4 & -361346.6 \\
\hline $063.1+13.9$ & NGC 6720 & 5335.1 & +330145.1 & $349.5+01.0$ & NGC 6302 & 171344.5 & -370611.6 \\
\hline $064.7+05.0$ & $\mathrm{BD}+303639$ & 193445.2 & +303059.2 & $352.6+00.1$ & PN H 1-12 & 172624.3 & -350141.8 \\
\hline $082.1+07.0$ & NGC 6884 & 201023.7 & +462740.0 & $352.8-00.2$ & PN H 1-13 & 172827.7 & -350730.4 \\
\hline $083.5+12.7$ & NGC 6826 & 194448.2 & +503131.3 & $358.5+02.6$ & PN HDW 8 & 173147.3 & -284203.5 \\
\hline $086.5-08.8$ & PN Hu 1-2 & 213308.2 & +393808.3 & $358.5+05.4$ & PN M 3-39 & 172111.5 & -271137.0 \\
\hline $089.0+00.3$ & NGC 7026 & 210618.7 & +475107.5 & $359.3-00.9$ & Pn HB 5 & 174756.3 & -295940.6 \\
\hline
\end{tabular}

we would obtain a direct measurement of the expected flux and not an extrapolation.

As an added value, the present work provides the first sizeable dataset of $43 \mathrm{GHz}$ measurements of PNe that constitute strong constraints to the observed SEDs in the very important spectral region where free-free emission and thermal dust emission may overlap. While interferometric high frequency observations provide us with detailed morphological information they quite often fail to entirely recover the extended emission. This eventually leads to underestimation of the total radio flux density. This problem is overcame by single-dish observations. The knowledge of the SEDs in the radio-millimetre spectral range is a crucial step for the study of the physics of dusty envelopes around PN. A correct evaluation of the free-free contribution, up to the millimetre range, when combined with information provided by far-IR observations, would allow us to determine the presence of an excess due to the existence of a cold dust component(s) (Gee et al. 1984; Hoare et al. 1992; Kemper et al. 2002) or of alternative emission mechanisms (Casassus et al. 2007). Since PNe and their progenitors are believed to be among the major sources of recycled interstellar matter, determining the properties of the dust ejected in the ISM is very important to the study of the Galaxy evolution in general.

\section{The $43 \mathrm{GHz}$ Noto survey}

\section{1. sample selection}

Our sample has been selected mainly from Condon \& Kaplan (1998), who performed a cross-correlation between the
Strasbourg-ESO Catalogue of Galactic Planetary Nebulae (Cat. $\langle\mathrm{V} / 84\rangle$, Acker et al. 1992) and the $1.4 \mathrm{GHz}$ NRAO VLA Sky Survey. Among these sources we selected only those whose flux densities at $1.4 \mathrm{GHz}$ (NVSS) is higher than $100 \mathrm{mJy}$, for a total of $64 \mathrm{PNe}$. In the conservative hypothesis of optically thin nebula at $1.4 \mathrm{GHz}$, a cut-off at $100 \mathrm{mJy}$ guarantees a $43 \mathrm{GHz}$ flux density easily detectable with the Noto Radiotelescope. This estimated $43 \mathrm{GHz}$ flux density will be a lower limit in the case of optically thick nebula at $1.4 \mathrm{GHz}$. We also considered Condon et al. (1999) who selected infrared PNs in NVSS by a crosscorrelation between NVSS and a sample from IRAS PSC on the basis of infrared colours characteristic of PNe. Only 3 of the 122 infrared PNs candidate have $S_{1.4 \mathrm{GHz}} \geq 100 \mathrm{mJy}$, yielding a final sample of 67 objects.

To avoid problems due to possible contamination in the Noto beam $\left(H P B W=54^{\prime \prime}\right)$, for each source of the sample, we have extract from NVSS a $25^{\prime} \times 25^{\prime}$ field, centred at the position of the target. Sources that show a very extended $\left(\geq 2^{\prime}\right)$ emission or that are located in very high confusion region have been rejected. This reduces our sample to 62 objects. The list of the selected targets, with names and positions, is reported in Table 1.

\subsection{Observations and results}

In the last few years the INAF-IRA $32 \mathrm{~m}$ Noto radiotelescope has undergone a series of structural improvements which have remarkably increased its capabilities as a single dish instrument. 
Table 2. Results. The columns are as following: source name (1); Noto measured flux density and its associated error (2 and 3); Gaussian source angular dimension as from NVSS (4); actual diameter of the source (5); total flux density corrected as explained in the text (6).

\begin{tabular}{|c|c|c|c|c|c|c|c|c|c|c|c|c|c|}
\hline $\begin{array}{l}\text { IAU Name } \\
\text { PN G }\end{array}$ & $\begin{array}{r}S_{43} \\
{[\mathrm{mJy}]}\end{array}$ & $\begin{array}{r}\sigma_{43} \\
{[\mathrm{mJy}]}\end{array}$ & $\begin{array}{l}\theta_{1.4} \\
{\left[{ }^{\prime \prime}\right]}\end{array}$ & $\begin{array}{r}\Theta \\
{\left[{ }^{\prime \prime}\right]}\end{array}$ & $\begin{array}{r}S_{43}^{(\mathrm{I})} \\
{[\mathrm{mJy}]}\end{array}$ & Ref.* & $\begin{array}{l}\text { IAU Name } \\
\text { PN G }\end{array}$ & $\begin{array}{r}S_{43} \\
{[\mathrm{mJy}]}\end{array}$ & $\begin{array}{r}\sigma_{43} \\
{[\mathrm{mJy}]}\end{array}$ & $\begin{array}{l}\theta_{1.4} \\
{\left[{ }^{\prime \prime}\right]}\end{array}$ & $\begin{array}{r}\Theta \\
{\left[{ }^{\prime \prime}\right]}\end{array}$ & $\begin{array}{r}S_{43}^{(\mathrm{c})} \\
{[\mathrm{mJy}]}\end{array}$ & Ref.* \\
\hline $000.3+12.2$ & $<180$ & 60 & 10 & - & - & 4,7 & $093.4+05.4$ & $<240$ & 80 & 49 & 72 & - & 2,1 \\
\hline $002.4+05.8$ & 1330 & 110 & 21 & 33 & 1530 & 4,7 & $093.5+01.4$ & 560 & 35 & 15 & 23 & 600 & 2,1 \\
\hline $003.1+02.9$ & 100 & 20 & 12 & - & 100 & 4,7 & $096.4+29.9$ & 400 & 70 & 12 & - & 420 & 2,1 \\
\hline $006.7-02.2$ & 300 & 90 & 21 & 33 & 350 & & $097.5+03.1$ & 230 & 25 & 33 & 51 & 320 & 2,1 \\
\hline $007.2+01.8$ & 310 & 50 & 11 & - & 320 & 4,7 & $106.5-17.6$ & 610 & 40 & 12 & - & 640 & 2,1 \\
\hline $008.0+03.9$ & 180 & 20 & 35 & 53 & 250 & 4,6 & $107.8+02.3$ & 280 & 25 & 16 & 26 & 310 & 2,1 \\
\hline 008.3-01.1 & 90 & 20 & 10 & - & 90 & 4,7 & $120.0+09.8$ & 420 & 70 & 28 & 43 & 530 & 2,1 \\
\hline 009.4-05.0 & 130 & 30 & 13 & 20 & 140 & 4,7 & $130.9-10.5$ & 140 & 40 & 59 & 85 & 310 & 2,1 \\
\hline $009.6+14.8$ & 270 & 60 & 14 & 23 & 290 & 4,7 & $138.8+02.8$ & $<150$ & 50 & 23 & 36 & - & 2,1 \\
\hline $010.1+00.7$ & 400 & 60 & 12 & - & 420 & $4,5,7$ & $144.5+06.5$ & 215 & 30 & 35 & 53 & 300 & 2,1 \\
\hline $010.8-01.8$ & 130 & 20 & 12 & - & 140 & 4,7 & $165.5-15.2$ & 220 & 30 & 94 & 129 & 890 & 2,1 \\
\hline 011.7-00.6 & $<150$ & 50 & 9 & - & - & 4,7 & $166.1+10.4$ & 100 & 30 & 9 & - & 100 & 2,1 \\
\hline 020.9-01.1 & 420 & 90 & 14 & 21 & 450 & 4 & $173.7+02.7$ & 260 & 20 & 12 & - & 270 & 1 \\
\hline $025.8-17.9$ & 270 & 30 & 15 & 23 & 290 & 4,7 & $194.2+02.5$ & 90 & 20 & 0 & - & 90 & $2,1,7$ \\
\hline $027.7+00.7$ & 110 & 20 & 0 & - & 110 & 3,6 & $197.8+17.3$ & 240 & 50 & 22 & 35 & 280 & $2,1,7$ \\
\hline $033.8-02.6$ & 290 & 50 & 15 & 23 & 310 & 1,7 & $206.4-40.5$ & 160 & 20 & 21 & 32 & 180 & 4,7 \\
\hline $034.6+11.8$ & 1220 & 100 & 12 & - & 1280 & $2,1,7$ & $215.2-24.2$ & 1100 & 100 & 0 & - & 1100 & 4,7 \\
\hline $035.1-00.7$ & 170 & 20 & 13 & 21 & 180 & 2,1 & $221.3-12.3$ & 350 & 50 & 11 & - & 360 & 4,7 \\
\hline $037.7-34.5$ & 375 & 40 & 15 & 24 & 400 & 4,7 & $234.8+02.4$ & 350 & 20 & 17 & 26 & 380 & 4,7 \\
\hline $039.8+02.1$ & 240 & 20 & 0 & - & 240 & 2,1 & $254.6+00.2$ & 1770 & 290 & 41 & 62 & 2800 & 5 \\
\hline $041.8-02.9$ & 230 & 20 & 78 & 109 & 710 & $2,1,7$ & $258.1-00.3$ & 180 & 40 & 5 & - & 180 & 8,7 \\
\hline $043.1+37.7$ & 230 & 30 & 11 & - & 240 & $2,1,7$ & $259.1+00.9$ & 270 & 60 & 42 & 63 & 430 & 8 \\
\hline $045.7-04.5$ & 140 & 20 & 25 & 40 & 170 & $2,3,1$ & $261.0+32.0$ & 290 & 30 & 20 & 31 & 330 & 4,7 \\
\hline $050.1+03.3$ & 140 & 30 & 41 & 62 & 220 & $2,1,7$ & $294.1+43.6$ & 130 & 20 & 47 & 70 & 230 & 4,7 \\
\hline $054.1-12.1$ & 130 & 40 & 9 & - & 130 & $2,1,7$ & $342.1+10.8$ & $<210$ & 70 & 33 & 50 & - & 7 \\
\hline $063.1+13.9$ & 255 & 75 & 48 & 71 & 460 & 2,1 & $349.5+01.0$ & 2150 & 220 & 15 & 23 & 2310 & $8,5,7$ \\
\hline $064.7+05.0$ & 565 & 20 & 11 & - & 590 & 2,1 & $352.6+00.1$ & 815 & 125 & 10 & - & 840 & 5 \\
\hline $082.1+07.0$ & 250 & 50 & 11 & - & 260 & 2,1 & $352.8-00.2$ & 420 & 50 & 15 & 23 & 450 & 5 \\
\hline $083.5+12.7$ & 320 & 40 & 16 & 25 & 350 & 2,1 & $358.5+02.6$ & $<120$ & 40 & 21 & 33 & - & 4,6 \\
\hline 086.5-08.8 & $<120$ & 40 & 10 & - & - & 2,1 & $358.5+05.4$ & 380 & 40 & 0 & - & 380 & 4,6 \\
\hline $089.0+00.3$ & 220 & 30 & 12 & - & 230 & 2,1 & $359.3-00.9$ & 290 & 70 & 0 & - & 290 & 5,7 \\
\hline
\end{tabular}

${ }^{*}$ References for 5 GHz measurements: 1) Becker et al. (1991); 2) Gregory et al. (1996); 3) Griffith et al. (1995); 4) Griffith et al. (1994); 5) Haynes et al. (1979); 6) Milne (1979); 7) Milne (1975); 8) Wright et al. (1994).

In particular, the installation of an active surface allows it to operate with good performance at high frequencies.

The observations reported in this paper were carried out in different epochs, between 2005 and 2006. The $43 \mathrm{GHz}$ superheterodyne receiver is a cooled double polarization receiver, with a typical zenith system temperature $\left(T_{\text {sys }}\right)$, in both channels, of the order of $80-100 \mathrm{~K}$, depending on the weather conditions. The gain ranges from 0.05 to $0.1 \mathrm{~K} / \mathrm{Jy}$ (Leto et al. 2006), depending on elevation, and this determines a zenith System Equivalent Flux Density (SEFD) of 1600-2000 Jy. The observations were performed with a $400 \mathrm{MHz}$ instantaneous band.

All the sources of our sample were observed with the on the fly (OTF) scan technique, which consists of driving the beam of the telescope across the source in RA direction. The typical scan duration was of the order of $20 \mathrm{~s}$, short enough to remain close to the white noise regime of the radiometer. In order to achieve a good signal to noise ratio, each source was observed many times, for a total integration time of $30 \mathrm{~min}$. Multiple OTF scans were then added reaching a typical $\mathrm{rms}$ of $2-3 \mathrm{mK}$.

Daily gain curves were obtained and the flux scale was fixed by using NGC 7027 as the primary calibrator and 3C 286 as the secondary calibrator. The adopted flux densities for NGC 7027 and 3C 286 are respectively 5.07 and $1.86 \mathrm{Jy}$. The assumed flux densities are those reported by Ott et al. (1994); in the case of
NGC 7027 the measure has been corrected for the observed decreament of 0.15 percent/year as reported by Perley et al. (2006).

We detected 55 out of the 62 observed objects, with a $89 \%$ detection rate. The results of such observations are reported in Table 2, where the $43 \mathrm{GHz}$ measured flux density, or its $3 \sigma$ upper limit, is listed (Col. 2). In the 4th and 10th columns of the same table we report the angular size at $1.4 \mathrm{GHz}$ of each source $\left(\theta_{1.4}\right)$, as derived from the analysis of the relative NVSS map, which has an angular resolution of $45^{\prime \prime}\left(\theta_{\text {NVSS }}\right)$. The source angular size has been derived as the geometrical mean of the minor and major axes obtained by fitting a two dimensional Gaussian at the source position in the map of the brightness distribution, and by performing a Gaussian deconvolution by means of the task JMFIT of the NRAO Astronomical Image Processing System (AIPS). In the case of partially resolved sources, this Gaussian angular diameter is related to the actual diameter of the source by a shape factor that accounts for the true structure of the source. Therefore, to compare the results from $1.4 \mathrm{GHz}$ data with those obtained in the optical, we need to correct the Gaussian angular diameters following van Hoof (2000). This correction has been applied only for sources with $\theta_{1.4} \geq 1 / 4 \theta_{\text {NVSS }}$, assuming a uniform density model; the obtained values are reported in Table $2(\Theta)$. Such radio derived values are generally in good agreement with those given by Tylenda et al. (2003), derived 


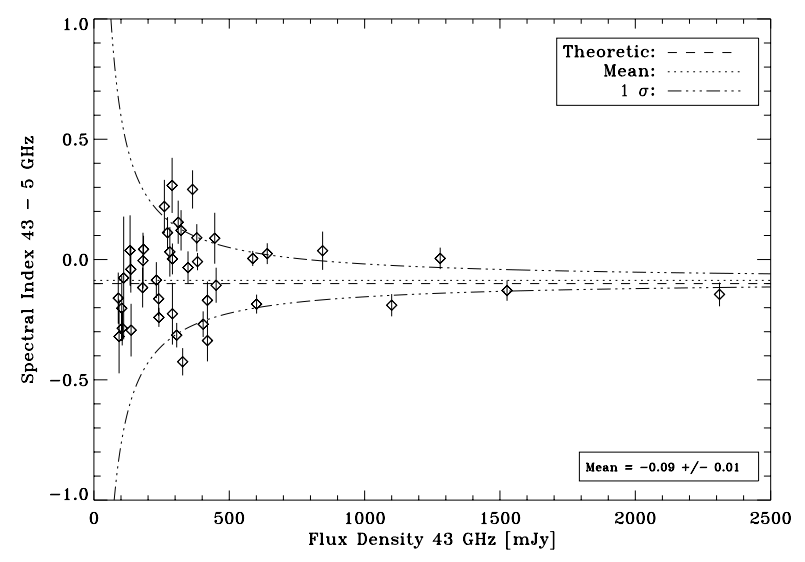

Fig. 1. Calculated 5-43 GHz spectral index as a function of flux at $43 \mathrm{GHz}$. As expected major dispersion is evident at lower fluxes.

from $\mathrm{H}_{\alpha}$ images, with a few exceptions for PNe with very complex optical morphologies (i.e. Hb5 and M3-39) or in the case of NGC 6302, where the radio source is much smaller than the optical one (Gomez et al. 1989).

To take into account possible partial resolution of the source by the Noto beam, assuming that the source size at $43 \mathrm{GHz}$ is the same as measured at $1.4 \mathrm{GHz}$, we corrected the measured flux density as

$S_{43}^{I}=S_{43} \times \frac{\theta_{\text {Noto }}^{2}+\theta_{1.4}^{2}}{\theta_{\text {Noto }}^{2}}$

where $\theta_{1.4}$ and $\theta_{\text {Noto }}$ are the Gaussian diameter of the source at $1.4 \mathrm{GHz}$ (reported in Table 2) and the width of the Noto radiotelescope beam $\left(H P B W=54^{\prime \prime}\right)$ respectively. Sources having an angular size greather than 25", comparable to the $43 \mathrm{GHz}$ Noto beam $(H P B W)$, have flux corrections that are significantly larger than the rms associated with the flux density. This means that they cannot be regarded as point-like. The resulting fluxes $S_{43}^{I}$ are listed in Table 2.

\section{The SEDs}

\subsection{The free-free contribution}

As a first step in our analysis, we derived the spectral index $\alpha$, $\left(S_{v} \propto v^{\alpha}\right)$, by combining the fluxes measured at $43 \mathrm{GHz}$ with the $5 \mathrm{GHz}$ single dish measurements available from the literature. To prevent any error due to resolving out some of the flux density, we considered only the objects that have angular size, at $1.4 \mathrm{GHz}$ $\left(\theta_{1.4 \mathrm{GHz}}\right)$, lower than $25^{\prime \prime}$. This reduces our sample of detected sources to $42 \mathrm{PNe}$ that can be considered point-like with respect to the telescope beam at the observational frequency. Literature $5 \mathrm{GHz}$ data are available only for 41 targets. The $5 \mathrm{GHz}$ single dish measurements are from surveys performed by the NRAO Green Bank $91 \mathrm{~m}$ telescope, with a beamwidth $(H P B W) \approx 3.5^{\prime}$ (Gregory et al. 1996; Becker et al. 1991) and Parke s 64 m telescope with a beamwidth $\approx 4.5^{\prime}$ (Griffith et al. 1995, 1994; Wright et al. 1994; Haynes et al. 1979; Milne 1979; Milme \& Aller 1975), as indicated in Table 2. For the sources observed in more than one survey, we used the average of the fluxes from the different measurements.

The spectral indices $\alpha$, calculated between the two frequencies, are shown in Fig. 1 as a function of the flux density at $43 \mathrm{GHz}$. Since its accuracy depends on the value of the measured flux densities and associated uncertainties, we computed the expected $1 \sigma$ as a function of the flux density, assuming a typical rms, associated with the $43 \mathrm{GHz}$ and $5 \mathrm{GHz}$ measurements, of $\sigma_{43 \mathrm{GHz}} \approx 50 \mathrm{mJy}$ and a $\sigma_{5 \mathrm{GHz}} \approx 35 \mathrm{mJy}$, respectively. The $\pm 1 \sigma$ uncertainty values around the mean value $\alpha=-0.09$ are shown in Fig. 1 as dot-dashed lines. For about $70 \%$ of the sources we obtain a spectral index inside the uncertainty lines, showing that it is statistically consistent with the value $\alpha=-0.1$, typical of an optically thin free-free emission. We conclude that for our sample, at least up to $43 \mathrm{GHz}$, the SED is dominated by the free-free emission and other contributions, if present, are negligible.

\subsection{The dust contribution}

PNs are usually surrounded by a dusty envelope which is the remnant of the precursor's wind. Dust thermally re-radiates the UV radiation of the central star determining an excess that may extend from the far-IR to the radio region. Such a contribution is typically of the order of $40 \%$ of the total flux from a PNe (Zhang \& Kwok 1991), being larger in young PNe, since, in more evolved PNe, the circumstellar material has already dispersed. We built the dust component of our sample of PNe by using IRAS measurements.

The IRAS data have been fitted by assuming that the dust emits as a blackbody modified by the frequency dependent dust opacity, that is $F_{v} \propto v^{p} B_{v}\left(T_{\mathrm{d}}\right)$, where $B_{v}\left(T_{\mathrm{d}}\right)$ is the Planck function for the dust temperature $T_{\mathrm{d}}$, and $p$ is the emissivity index. The index $p$ strongly depends on the mineralogical composition of the grain and on their physical shape. In our calculation, following the results from detailed modelling of dust emission from PNe (Hoare 1990), we assumed, for the slope of the far-IR emissivity law, a typical value of $p=1.5$. Because of the strong contamination by line emission of the $12 \mu \mathrm{m}$ IRAS band (Stasinska \& Szczerba 1999) only 25, 60 and $100 \mu$ m fluxes have been used in the fitting procedure, using only fluxes with a quality code better than 1 .

\section{Estimates of flux density in Planck channels}

The SEDs with both ionized gas and the dust contribution, evaluated in Sects. 3.1 and 3.2, were built for all the targets in our sample. In Fig. 2, four such SEDs are shown as an example. In the SEDs, between the radio and far-IR there is a large gap where measurements are missing. New millimetre and sub-millimetre observations are clearly necessary to better characterize and constrain the emission from different components, where the radio flux, from the ionized fraction of the nebula, could be merged with the dust contribution. This gap will be partially filled by the ESA-PLANCK mission, as the satellite is equipped with a Low Frequency Instrument (LFI), operating at 30, 44 and $70 \mathrm{GHz}$, and a High Frequency Instrument (HFI), operating at 100, 143, $217,353,545$ and $857 \mathrm{GHz}$.

In order to evaluate the possibility that PLANCK will actually detect sources in our sample, we should compare, for each observational band, the expected flux from the sources to the foreseen sensitivity. The expected fluxes are derived by summing, in each PLANCK channel, both the dust and ionized gas contribution. The first has been obtained by extrapolating the modified blackbody fit (dashed lines in Fig. 2); the latter has been obtained from fitting free-free emission from an optically thin nebula to the $5 \mathrm{GHz}\left(6 \times 10^{4} \mu \mathrm{m}\right)$ and $43 \mathrm{GHz}\left(7 \times 10^{3} \mu \mathrm{m}\right)$ data points and then extrapolating to the Planck channels (dotted line in Fig. 2), the NVSS measurements at $1.4 \mathrm{GHz}\left(2 \times 10^{5} \mu \mathrm{m}\right)$ have been also displayed. 

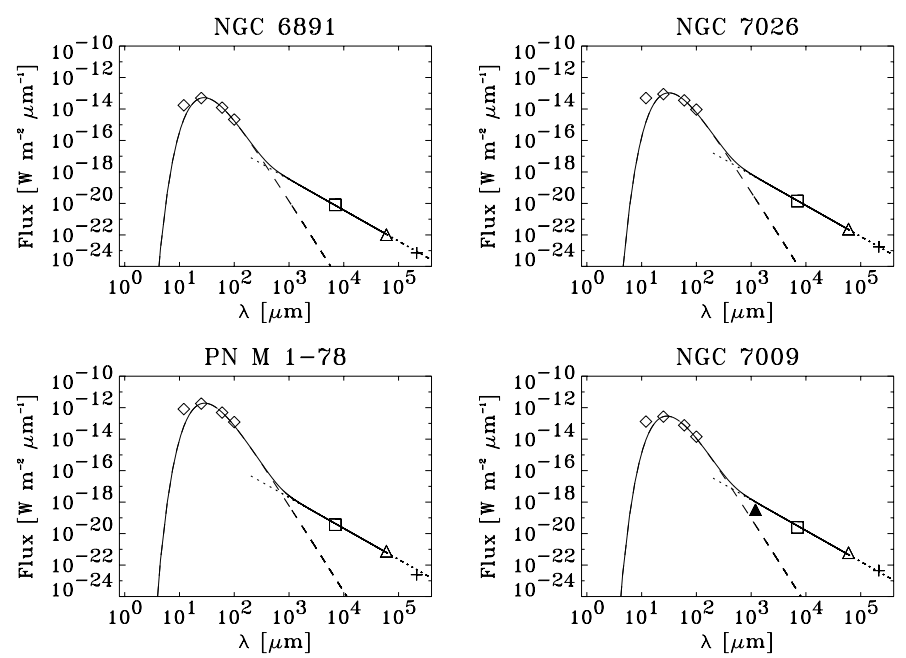

Fig. 2. Spectral Energy Distributions of four of our targets. Both ionized gas (dotted line) and thermal dust (dashed line) contributions are shown. The continuous line is the total emission. Diamonds: IRAS data; squares: our measurements at $43 \mathrm{GHz}\left(7 \times 10^{3} \mu \mathrm{m}\right)$; triangle: literature measures at $5 \mathrm{GHz}\left(6 \times 10^{4} \mu \mathrm{m}\right)$; cross: NVSS data at $1.4 \mathrm{GHz}(2 \times$ $10^{5} \mu \mathrm{m}$ ); filled triangle: $250 \mathrm{GHz}$ datum from Casassus et al. (2007).

Following Umana et al. (2006), the foreseen total sensitivity is assumed to be the sum in quadrature of all the sources of confusion noise, namely the nominal instrumental Planck sensitivity per resolution element, the Galactic and extragalactic foregrounds confusion noise (Toffolatti et al. 1998), the CMB confusion noise (Bennett et al. 2003). All the considered confusion sources will contribute to a total sensitivity $(\sigma)$ ranging from $270 \mathrm{mJy}$ for the $30 \mathrm{GHz}$ channel to $220 \mathrm{mJy}$ for the $857 \mathrm{GHz}$ channel. In Fig. 3 we show the percentage of the objects from our sample that could be detected over the $3 \sigma$ threshold for each channel of the PLANCK instruments. A small number of PNe belonging to our sample will be detected by PLANCK in the radio (LFI channels), while most of our targets will be easily seen by PLANCK in the millimetre and sub-millimetre bands (HFI channels).

Recently 17 sources in our sample were observed by Casassus et al. (2007) at 250 and/or $31 \mathrm{GHz}$. While the results of the measurements at $31 \mathrm{GHz}$ (15 sources) agree quite well with our estimates, the $250 \mathrm{GHz}$ measured fluxes (10 sources) appeared to be systematically lower, on average by a factor of 2 , than our free-free extrapolations (see the SED of NGC 7009 in Fig. 2 as an example). Understanding whether the differences between our estimates and the Casassus et al. (2007) measurements are due to the sources being partially resolved at that frequency or to some intrinsic properties of the source, is beyond the scope of this paper. Casassus et al. (2007) claim that such an unexpected drop in the measured flux densities could be attributed to a synchrotron component absorbed by a cold nebular screen, but they also speculate about other possible emission/absorption mechanisms which may operate in the ionized part of the nebula, without reaching any firm conclusions. If we conservatively assume that such a peculiarity is present in all the PNe of our sample, the flux estimates in the $217 \mathrm{GHz}$ PLANCK band has to be reduced by a factor of 2 and the expected detection rate in the same band reduces to about $20 \%$. The estimates in the higher frequency bands remain unaffected due to the predominance of the thermal dust emission. We foresee an important contribution of the PLANCK mission also to the study of PNe, as it would

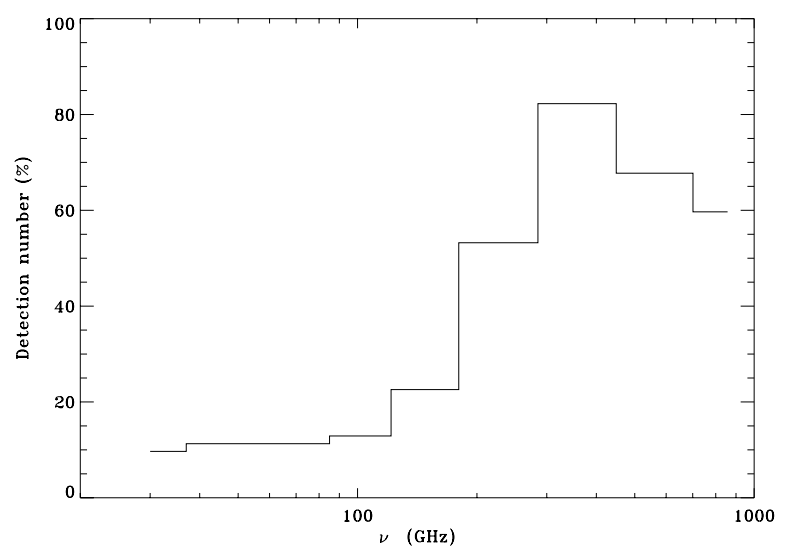

Fig. 3. Histogram showing the fraction of planetary nebulae belonging to our sample that can be detected by the Planck satellite.

provide measurements in a very important, but still poorly explored, spectral region.

\section{Summary}

We have presented new $7 \mathrm{~mm}(43 \mathrm{GHz})$ observations of a sample of radio-bright PNe, carried out with the INAF-IRA Noto Radiotelescope. Such observations have been used to derive the high-frequency free-free contribution, due to the ionized fraction of the circumstellar envelope. So far, the majority of high frequency radio observations of $\mathrm{PNe}$ have been conducted with interferometers, that reveal details of source radio morphology but could, in principle, resolve out some of the extended emission. Our single dish measurements provide an extended database of millimetre observations of PNe to be used when is necessary to know the overall emission such as when building a SED. We used our measurements that trace the free-free contribution together with IRAS measurements, which trace the thermal dust emission, to build up the observed SED from radio to far-IR and by extrapolation to estimate the expected fluxes in the spectral range between radio and sub-mm, where observational data are missing.

When comparing the expected millimetre-sub-millimetre fluxes with the total foreseen sensitivity of the forthcoming ESA PLANCK mission we estimate that a consistent number of our targets will be easily detected by PLANCK, mostly at higher frequency channels. Therefore, even if designed for cosmological study, PLANCK could also contribute to PNe investigation. Results from such kinds of observations, once modelled with the appropriate code (i.e. CLOUDY), would provide important constraints on the chemical composition and structure of the CSEs. The analysis of the modelled SEDs could point out the presence of multi-shells, related to multiple mass-loss event of the central object during its previous evolution (AGB), or a contribution of different emission processes, besides free-free and thermal emission from dust, as recently claimed by Casassus et al. (2007). Moreover, PLANCK results can be considered as pathfinders for future instrumentation, with the same frequency coverage, such as ALMA, as they will help in planning more focused experiments aimed to investigate the morphological details of the sources.

Acknowledgements. Based on obervations with the Noto Telescope operated by INAF-Istituto di Radioastronomia This work has been partially supported by ASI through contract Planck LFI Activity of Phase E2. 


\section{References}

Acker, A., Ochsenbein, F., Stenholm, B., et al. 1992, ESOPN, 1,

Becker, R. H., White, R. L., \& Edwards, A. L. 1991, ApJS, 75, 1

Bennett, C. L. 2003, ApJS, 148, 1

Casassus, S., Nyman, L. A., Dickinson, C., \& Pearson, T. J. 2007, MNRAS, accepted [arXiv:0708.2385]

Condon, J. J., \& Kaplan, D. L. 1998, ApJS, 117, 361

Condon, J. J., Kaplan, D. L., \& Terzian, Y. 1999, ApJS, 123, 219

Gee, G., Emerson, J. P., Ade, P. A. R., Robson, E. I., \& Nolt, I. G. 1984, MNRAS, 208,517

Gomez, Y., Moran, J. M., Rodriguez, L. F., \& Gray, G. 1989, ApJ, 345, 862

Gregory, P. C., Scott, W. K., Douglas, K., \& Condon, J. J. 1996, ApJS, 103, 427

Griffith, M. R., Wright, A. E., Burke, B. F., \& Ekers, R. D. 1994, ApJS, 90, 179

Griffith, M. R., Wright, A. E., Burke, B. F., \& Ekers, R. D. 1995, ApJS, 97, 347

Haynes, R. F., Caswell, J. L., \& Simons, L. W. J. 1979, AuJPA, 48, 1

Hoare, M. G. 1990, MNRAS, 244, 193
Hoare, M. G., Roche, P. F., \& Clegg, R. E. S. 1992, MNRAS, 258, 257

Leto, P., Buemi, C. S., Trigilio, C., et al. 2006, MSAIS, 10, 87

Luo, S. G., Condon, J. J., \& Yin, Q. F. 2005, ApJS, 159, 282

Kemper, F., Molster, F. J., Jager, C., \& Waters, L. B. F. M. 2002, A\&A, 394, 679

Milne, D. K. 1979, A\&AS, 36, 227

Milne, D. K., \& Aller, L. H. 1975, A\&A, 38, 183

Ott, M., Witzel, A., Quirrenbach, A., et al. 1994, A\&A, 284, 331

Perley, R. A., Zijlstra, A., \& van Hoof, P. 2006, A\&AS, 209, 9202

Siodmiak, N., \& Tylenda, R. 2001, A\&A, 373, 1032

Stasinska, G., \& Szczerba, R. 1999, A\&A, 352, 297

Tylenda, R., Siodmiak, N., Gorny, S. K., Corradi, R. L. M., \& Schwarz, H. E. 2003, A\&A, 405, 627

Toffolatti, L., Argueso Gomez, F., de Zotti, G., et al. 1998, MNRAS, 297, 117

Umana, G., Burigana, C., \& Trigilio, C. 2006, MSAIS, 9, 279

van Hoof, P. A. M. 2000, MNRAS, 314, 99

Wright, A. E., Griffith, M. R., Burke, B. F., \& Ekers, R. D. 1994, ApJS, 91, 111 Zhang, C. Y., \& Kwok, S. 1991, A\&A, 250, 179 